\title{
A note on geometrically convex functions
}

\author{
Muhamet Emin Özdemir ${ }^{1}$, Çetin Yildiz ${ }^{1 *}$ and Mustafa Gürbüz ${ }^{2}$
}

${ }^{\text {*Correspondence: }}$

cetin@atauni.edu.tr

${ }^{1}$ Department of Mathematics,

K. K. Education Faculty, Atatürk

University, Campus, Erzurum, 25240,

Turkey

Full list of author information is

available at the end of the article

\begin{abstract}
In this paper, we establish several new inequalities for twice differentiable mappings that are connected with the celebrated Hermite-Hadamard integral inequality.

MSC: Primary 26D15; secondary 26A51

Keywords: Hermite-Hadamard inequality; geometrically convex function; $(\alpha, m)$-geometrically convex function
\end{abstract}

\section{Historical background and introduction}

The following double inequality is well known in the literature as Hadamard's inequality:

Let $f: I \subseteq \mathbb{R} \rightarrow \mathbb{R}$ be a convex function defined on an interval $I$ of real numbers, $a, b \in I$ and $a<b$, we have

$$
f\left(\frac{a+b}{2}\right) \leq \frac{1}{b-a} \int_{a}^{b} f(x) d x \leq \frac{f(a)+f(b)}{2}
$$

Both inequalities hold in the reversed direction if $f$ is concave.

It was first discovered by Hermite in 1881 in the Journal Mathesis (see [1]). Inequality (1.1) was nowhere mentioned in the mathematical literature until 1893. Beckenbach, a leading expert on the theory of convex functions, wrote that inequality (1.1) was proven by Hadamard in 1893 (see [2]). In 1974 Mitrinovič found Hermite's note in Mathesis. That is why, inequality (1.1) was known as the Hermite-Hadamard inequality.

A function $f:[a, b] \subset \mathbb{R} \rightarrow \mathbb{R}$ is said to be convex if whenever $x, y \in[a, b]$ and $t \in[0,1]$, the following inequality holds:

$$
f(t x+(1-t) y) \leq t f(x)+(1-t) f(y)
$$

We say that $f$ is concave if $(-f)$ is convex. This definition has its origins in Jensen's results from [3] and has opened up the most extended, useful and multi-disciplinary domain of mathematics, namely, convex analysis. Convex curves and convex bodies have appeared in mathematical literature since antiquity and there are many important results related to them.

In [4], Miheşan introduced the class of $(\alpha, m)$-convex functions in the following way: The function $f:[0, b] \rightarrow \mathbb{R}$ is said to be $(\alpha, m)$-convex, where $(\alpha, m) \in[0,1]^{2}$, if for every $x, y \in[0, b]$ and $t \in[0,1]$, we have

$$
f(t x+m(1-t) y) \leq t^{\alpha} f(x)+m\left(1-t^{\alpha}\right) f(y)
$$

This class is usually denoted by $K_{m}^{\alpha}(b)$.

\section{Springer}

(c)2014 Özdemir et al.; licensee Springer. This is an Open Access article distributed under the terms of the Creative Commons Attribution License (http://creativecommons.org/licenses/by/2.0), which permits unrestricted use, distribution, and reproduction in any medium, provided the original work is properly cited. 
In [5], the concept of geometrically convex functions was introduced as follows.

Definition 1 A function $f: I \subset \mathbb{R}_{+} \rightarrow \mathbb{R}_{+}$is said to be a geometrically convex function if

$$
f\left(x^{t} y^{1-t}\right) \leq[f(x)]^{t}[f(y)]^{1-t}
$$

for all $x, y \in I$ and $t \in[0,1]$.

In [6], the definition of $m$ - and $(\alpha, m)$-geometric convexity was introduced as follows.

Definition 2 Let $f(x)$ be a positive function on $[0, b]$ and $m \in(0,1]$. If

$$
f\left(x^{t} y^{m(1-t)}\right) \leq[f(x)]^{t}[f(y)]^{m(1-t)}
$$

holds for all $x, y \in[0, b]$ and $t \in[0,1]$, then we say that the function $f(x)$ is $m$-geometrically convex on $[0, b]$.

It is clear that when $m=1, m$-geometrically convex functions become geometrically convex functions.

Definition 3 Let $f(x)$ be a positive function on $[0, b]$ and $(\alpha, m) \in(0,1] \times(0,1]$. If

$$
f\left(x^{t} y^{m(1-t)}\right) \leq[f(x)]^{t^{\alpha}}[f(y)]^{m\left(1-t^{\alpha}\right)}
$$

holds for all $x, y \in[0, b]$ and $t \in[0,1]$, then we say that the function $f(x)$ is $(\alpha, m)$ geometrically convex on $[0, b]$.

If $\alpha=m=1$, the $(\alpha, m)$-geometrically convex function becomes a geometrically convex function on $[0, b]$.

Lemma 1 For $x, y \in[0, \infty)$ and $m, t \in(0,1]$, if $x<y$ and $y \geq 1$, then

$$
x^{t} y^{m(1-t)} \leq t x+(1-t) y .
$$

For some recent results connected with geometrically convex functions, see [5-9].

Definition 4 Let $a, b \in \mathbb{R}, a, b \neq 0$ and $|a| \neq|b|$. Logarithmic mean for real numbers was introduced as follows:

$$
L(a, b)=\frac{a-b}{\ln |a|-\ln |b|} .
$$

Theorem 1 (see [10]) Let $f, g:[a, b] \rightarrow \mathbb{R}$ be integrable functions, both increasing or both decreasing. Furthermore, let $p:[a, b] \rightarrow \mathbb{R}_{+}$be an integrable function. Then

$$
\int_{a}^{b} p(x) f(x) d x \int_{a}^{b} p(x) g(x) d x \leq \int_{a}^{b} p(x) d x \int_{a}^{b} p(x) f(x) g(x) d x .
$$

If one of the functions $f$ or $g$ is nonincreasing and the other nondecreasing, then the inequality in (1.3) is reversed. Inequality (1.3) is known in the literature as the Chebyshev inequality 
and so are the following special cases of (1.3):

$$
\frac{1}{b-a} \int_{a}^{b} f(x) d x \int_{a}^{b} g(x) d x \leq \int_{a}^{b} f(x) g(x) d x
$$

and

$$
\int_{0}^{1} f(x) d x \int_{0}^{1} g(x) d x \leq \int_{0}^{1} f(x) g(x) d x .
$$

In order to prove our main results, we need the following lemma (see [11]).

Lemma 2 Let $f: I \subset \mathbb{R} \rightarrow \mathbb{R}$ be a twice differentiable function on $I^{\circ}, a, b \in I$ with $a<b$ and $f^{\prime \prime} \in L_{1}[a, b]$. Then the following equality holds:

$$
\frac{f(a)+f(b)}{2}-\frac{1}{b-a} \int_{a}^{b} f(x) d x=\frac{(b-a)^{2}}{2} \int_{0}^{1} t(1-t) f^{\prime \prime}(t a+(1-t) b) d t .
$$

In [12], some inequalities of Hermite-Hadamard type for differentiable convex mappings were proven using the following lemma.

Lemma 3 Let $f: I \subset \mathbb{R} \rightarrow \mathbb{R}$ be a twice differentiable function on $I^{\circ}, a, b \in I^{\circ}$ with $a<b$. If $f^{\prime \prime} \in L_{1}[a, b]$, then

$$
\begin{aligned}
& \frac{1}{b-a} \int_{a}^{b} f(x) d x-f\left(\frac{a+b}{2}\right) \\
& =\frac{(b-a)^{2}}{4} \int_{0}^{1} m(t)\left[f^{\prime \prime}(t a+(1-t) b)+f^{\prime \prime}((1-t) a+t b)\right] d t,
\end{aligned}
$$

where

$$
m(t)= \begin{cases}t^{2}, & t \in\left[0, \frac{1}{2}\right) \\ (1-t)^{2}, & t \in\left[\frac{1}{2}, 1\right] .\end{cases}
$$

In this paper, we establish some integral inequalities of Hermite-Hadamard type related to geometrically convex functions and $(\alpha, m)$-geometrically convex functions.

\section{Results for geometrically convex functions}

We will establish some new results connected with the right-hand side of (1.1).

Theorem 2 Let $f: I \subset \mathbb{R}_{+} \rightarrow \mathbb{R}_{+}$be a twice differentiable function on $I^{\circ}, a, b \in I$ with $a<b$ and $f^{\prime \prime} \in L_{1}[a, b]$. If $\left|f^{\prime \prime}\right|^{q}$ is geometrically convex and monotonically decreasing on $[a, b]$ for $p>1$ and $t \in[0,1]$, then we have

$$
\begin{aligned}
& \left|\frac{f(a)+f(b)}{2}-\frac{1}{b-a} \int_{a}^{b} f(x) d x\right| \\
& \leq \frac{(b-a)^{2}}{2}\left(\frac{2^{-1-2 p} \sqrt{\pi} \Gamma(1+p)}{\Gamma\left(\frac{3}{2}+p\right)}\right)^{\frac{1}{p}}\left\{L\left(\left|f^{\prime \prime}(a)\right|^{q},\left|f^{\prime \prime}(b)\right|^{q}\right)\right\}^{\frac{1}{q}},
\end{aligned}
$$

where $\frac{1}{p}+\frac{1}{q}=1$. 
Proof From Lemma 2 with the properties of modulus and using the Hölder inequality, we have

$$
\begin{aligned}
& \left|\frac{f(a)+f(b)}{2}-\frac{1}{b-a} \int_{a}^{b} f(x) d x\right| \\
& \quad \leq \frac{(b-a)^{2}}{2} \int_{0}^{1} t(1-t)\left|f^{\prime \prime}(t a+(1-t) b)\right| d t \\
& \quad \leq \frac{(b-a)^{2}}{2}\left(\int_{0}^{1}\left(t-t^{2}\right)^{p} d t\right)^{\frac{1}{p}}\left(\int_{0}^{1}\left|f^{\prime \prime}(t a+(1-t) b)\right|^{q} d t\right)^{\frac{1}{q}} \\
& \quad=\frac{(b-a)^{2}}{2}\left(\frac{2^{-1-2 p} \sqrt{\pi} \Gamma(1+p)}{\Gamma\left(\frac{3}{2}+p\right)}\right)^{\frac{1}{p}}\left(\int_{0}^{1}\left|f^{\prime \prime}(t a+(1-t) b)\right|^{q} d t\right)^{\frac{1}{q}} .
\end{aligned}
$$

We used the beta and gamma functions to evaluate the integral

$$
\begin{aligned}
& \int_{0}^{1}\left(t-t^{2}\right)^{p} d t=\int_{0}^{1} t^{p}(1-t)^{p} d t=\beta(p+1, p+1) \\
& \beta(x, x)=2^{1-2 x} \beta\left(\frac{1}{2}, x\right) \quad \text { and } \quad \beta(x, y)=\frac{\Gamma(x) \Gamma(y)}{\Gamma(x+y)} .
\end{aligned}
$$

Thus, we have

$$
\beta(p+1, p+1)=2^{1-2(p+1)} \frac{\Gamma\left(\frac{1}{2}\right) \Gamma(p+1)}{\Gamma\left(\frac{3}{2}+p\right)},
$$

where $\Gamma\left(\frac{1}{2}\right)=\sqrt{\pi}$.

Since $\left|f^{\prime \prime}\right|^{q}$ is geometrically convex and monotonically decreasing on $[a, b]$, we obtain

$$
\begin{aligned}
& a^{t} b^{1-t} \leq t a+(1-t) b, \\
& \left|f^{\prime \prime}(t a+(1-t) b)\right|^{q} \leq\left|f^{\prime \prime}\left(a^{t} b^{1-t}\right)\right|^{q} .
\end{aligned}
$$

Therefore, we have

$$
\begin{aligned}
I & =\int_{0}^{1}\left|f^{\prime \prime}(t a+(1-t) b)\right|^{q} d t \\
& \leq \int_{0}^{1}\left|f^{\prime \prime}\left(a^{t} b^{1-t}\right)\right|^{q} d t \\
& \leq \int_{0}^{1}\left[\left|f^{\prime \prime}(a)\right|^{t}\left|f^{\prime \prime}(b)\right|^{1-t}\right]^{q} d t \\
& =L\left(\left|f^{\prime \prime}(a)\right|^{q},\left|f^{\prime \prime}(b)\right|^{q}\right) .
\end{aligned}
$$

By making use of inequalities (2.3) and (2.4) in (2.2), we obtain (2.1). This completes the proof.

Theorem 3 Let $f: I \subset \mathbb{R}_{+} \rightarrow \mathbb{R}_{+}$be a twice differentiable function on $I^{\circ}, a, b \in I$ with $a<b$ and $f^{\prime \prime} \in L_{1}[a, b]$. If $\left|f^{\prime \prime}\right|^{q}$ is geometrically convex and monotonically decreasing on $[a, b]$ for 
$q \geq 1$ and $t \in[0,1]$, then the following inequality holds:

$$
\left|\frac{f(a)+f(b)}{2}-\frac{1}{b-a} \int_{a}^{b} f(x) d x\right| \leq \frac{(b-a)^{2}}{2}\left(\frac{1}{6}\right)^{\frac{1}{p}}\left|f^{\prime \prime}(b)\right| \cdot N(t, q),
$$

where

$$
N(t, q)=\left(\int_{0}^{1} t(1-t)\left(\frac{\left|f^{\prime \prime}(a)\right|}{\left|f^{\prime \prime}(b)\right|}\right)^{q t} d t\right)^{\frac{1}{q}} .
$$

Proof From Lemma 2 and using the well-known power-mean inequality, we have

$$
\begin{aligned}
& \left|\frac{f(a)+f(b)}{2}-\frac{1}{b-a} \int_{a}^{b} f(x) d x\right| \\
& \quad \leq \frac{(b-a)^{2}}{2} \int_{0}^{1} t(1-t)\left|f^{\prime \prime}(t a+(1-t) b)\right| d t \\
& \quad \leq \frac{(b-a)^{2}}{2}\left(\int_{0}^{1} t(1-t) d t\right)^{1-\frac{1}{q}}\left(\int_{0}^{1} t(1-t)\left|f^{\prime \prime}(t a+(1-t) b)\right|^{q} d t\right)^{\frac{1}{q}} \\
& \quad=\frac{(b-a)^{2}}{2}\left(\frac{1}{6}\right)^{1-\frac{1}{q}}\left(\int_{0}^{1} t(1-t)\left|f^{\prime \prime}(t a+(1-t) b)\right|^{q} d t\right)^{\frac{1}{q}} .
\end{aligned}
$$

Since $\left|f^{\prime \prime}\right|^{q}$ is geometrically convex and monotonically decreasing on $[a, b]$, we have

$$
\begin{aligned}
& \left|\frac{f(a)+f(b)}{2}-\frac{1}{b-a} \int_{a}^{b} f(x) d x\right| \\
& \quad \leq \frac{(b-a)^{2}}{2}\left(\frac{1}{6}\right)^{1-\frac{1}{q}}\left(\int_{0}^{1} t(1-t)\left[\left|f^{\prime \prime}(a)\right|^{t}\left|f^{\prime \prime}(b)\right|^{1-t}\right]^{q} d t\right)^{\frac{1}{q}} \\
& =\frac{(b-a)^{2}}{2}\left(\frac{1}{6}\right)^{1-\frac{1}{q}}\left|f^{\prime \prime}(b)\right|\left(\int_{0}^{1} t(1-t)\left(\frac{\left|f^{\prime \prime}(a)\right|}{\left|f^{\prime \prime}(b)\right|}\right)^{q t} d t\right)^{\frac{1}{q}},
\end{aligned}
$$

which completes the proof.

Corollary 1 In Theorem 3 , since $\left(\frac{1}{6}\right)^{\frac{1}{p}}<1$, for $p>1$, we have

$$
\left|\frac{f(a)+f(b)}{2}-\frac{1}{b-a} \int_{a}^{b} f(x) d x\right| \leq \frac{(b-a)^{2}}{2}\left|f^{\prime \prime}(b)\right| N(t, q)
$$

Now, we will establish some new results connected with the left-hand side of (1.1).

Theorem 4 Let $f: I \subset \mathbb{R}_{+} \rightarrow \mathbb{R}_{+}$be a twice differentiable function on $I^{\circ}, a, b \in I$ with $a<b$ and $f^{\prime \prime} \in L_{1}[a, b]$. If $\left|f^{\prime \prime}\right|^{q}$ is geometrically convex and monotonically decreasing on $[a, b]$ and $t \in[0,1]$, then we have the following inequality:

$$
\left|\frac{1}{b-a} \int_{a}^{b} f(x) d x-f\left(\frac{a+b}{2}\right)\right| \leq \frac{(b-a)^{2}}{8(2 p+1)^{\frac{1}{p}}}\left\{L\left(\left|f^{\prime \prime}(a)\right|^{q},\left|f^{\prime \prime}(b)\right|^{q}\right)\right\}^{\frac{1}{q}},
$$

where $\frac{1}{p}+\frac{1}{q}=1$. 
Proof From Lemma 3 and using the well-known Hölder integral inequality, we get

$$
\begin{aligned}
& \left|\frac{1}{b-a} \int_{a}^{b} f(x) d x-f\left(\frac{a+b}{2}\right)\right| \\
& \leq \frac{(b-a)^{2}}{4}\left(\int_{0}^{1}|m(t)|^{p} d t\right)^{\frac{1}{p}}\left\{\left(\int_{0}^{1}\left|f^{\prime \prime}(t a+(1-t) b)\right|^{q} d t\right)^{\frac{1}{q}}\right. \\
& \left.\quad+\left(\int_{0}^{1}\left|f^{\prime \prime}((1-t) a+t b)\right|^{q} d t\right)^{\frac{1}{q}}\right\}
\end{aligned}
$$

Since $\left|f^{\prime \prime}\right|^{q}$ is geometrically convex and monotonically decreasing on $[a, b]$, we know that for $t \in[0,1]$,

$$
\begin{aligned}
\left|f^{\prime \prime}(t a+(1-t) b)\right|^{q} & \leq\left|f^{\prime \prime}\left(a^{t} b^{1-t}\right)\right|^{q} \\
& \leq\left\{\left|f^{\prime \prime}(a)\right|^{t}\left|f^{\prime \prime}(b)\right|^{1-t}\right\}^{q}
\end{aligned}
$$

Hence

$$
\begin{aligned}
& \left|\frac{1}{b-a} \int_{a}^{b} f(x) d x-f\left(\frac{a+b}{2}\right)\right| \\
& \quad \leq \frac{(b-a)^{2}}{16(2 p+1)^{\frac{1}{p}}}\left\{\left[\int_{0}^{1}\left(\left|f^{\prime \prime}(a)\right|^{t}\left|f^{\prime \prime}(b)\right|^{1-t}\right)^{q} d t\right]^{\frac{1}{q}}+\left[\int_{0}^{1}\left(\left|f^{\prime \prime}(a)\right|^{1-t}\left|f^{\prime \prime}(b)\right|^{t}\right)^{q} d t\right]^{\frac{1}{q}}\right\} \\
& \quad=\frac{(b-a)^{2}}{8(2 p+1)^{\frac{1}{p}}}\left\{L\left(\left|f^{\prime \prime}(a)\right|^{q},\left|f^{\prime \prime}(b)\right|^{q}\right)\right\}^{\frac{1}{q}},
\end{aligned}
$$

where we have used the fact that

$$
\int_{0}^{1}|m(t)|^{p} d t=\int_{0}^{\frac{1}{2}} t^{2 p} d t+\int_{\frac{1}{2}}^{1}(1-t)^{2 p} d t=\frac{1}{4 p(2 p+1)}
$$

which completes the proof.

Corollary 2 In Theorem 4, since $\frac{1}{(2 p+1)^{\frac{1}{p}}}<1$, for $p>1$, we have

$$
\left|\frac{1}{b-a} \int_{a}^{b} f(x) d x-f\left(\frac{a+b}{2}\right)\right| \leq \frac{(b-a)^{2}}{8}\left\{L\left(\left|f^{\prime \prime}(a)\right|^{q},\left|f^{\prime \prime}(b)\right|^{q}\right)\right\}^{\frac{1}{q}} .
$$

Theorem 5 Let $f: I^{\circ} \subset \mathbb{R}_{+} \rightarrow \mathbb{R}_{+}$be a twice differentiable function on $I^{\circ}, a, b \in I$ with $a<b$ and $f^{\prime \prime} \in L_{1}[a, b]$. If $\left|f^{\prime \prime}\right|^{q}$ is geometrically convex and monotonically decreasing on $[a, b]$ and $q \geq 1$, then we have

$$
\left|\frac{1}{b-a} \int_{a}^{b} f(x) d x-f\left(\frac{a+b}{2}\right)\right| \leq \frac{(b-a)^{2}}{4}\left(\frac{1}{12}\right)^{\frac{1}{p}}\left\{\left|f^{\prime \prime}(a)\right| \cdot A(t, q)+\left|f^{\prime \prime}(b)\right| \cdot B(t, q)\right\}
$$

where

$$
A(t, q)=\left(\int_{0}^{1}|m(t)| \cdot\left(\frac{\left|f^{\prime \prime}(b)\right|}{\left|f^{\prime \prime}(a)\right|}\right)^{q t} d t\right)^{\frac{1}{q}}, \quad B(t, q)=\left(\int_{0}^{1}|m(t)| \cdot\left(\frac{\left|f^{\prime \prime}(a)\right|}{\left|f^{\prime \prime}(b)\right|}\right)^{q t} d t\right)^{\frac{1}{q}}
$$


Proof From Lemma 3 and using the well-known power-mean inequality, we get

$$
\begin{aligned}
& \left|\frac{1}{b-a} \int_{a}^{b} f(x) d x-f\left(\frac{a+b}{2}\right)\right| \\
& \leq \frac{(b-a)^{2}}{4}\left(\int_{0}^{1}|m(t)| d t\right)^{\frac{1}{p}}\left\{\left(\int_{0}^{1}|m(t)|\left|f^{\prime \prime}(t a+(1-t) b)\right|^{q} d t\right)^{\frac{1}{q}}\right. \\
& \left.\quad+\left(\int_{0}^{1}|m(t)|\left|f^{\prime \prime}((1-t) a+t b)\right|^{q} d t\right)^{\frac{1}{q}}\right\} .
\end{aligned}
$$

Since $\left|f^{\prime \prime}\right|^{q}$ is geometrically convex and monotonically decreasing on $[a, b]$, we have

$$
\begin{aligned}
& \left|\frac{1}{b-a} \int_{a}^{b} f(x) d x-f\left(\frac{a+b}{2}\right)\right| \\
& \leq \frac{(b-a)^{2}}{4(12)^{\frac{1}{p}}}\left\{\left(\int_{0}^{1}|m(t)|\left[\left|f^{\prime \prime}(a)\right|^{t}\left|f^{\prime \prime}(b)\right|^{1-t}\right]^{q} d t\right)^{\frac{1}{q}}\right. \\
& \left.\quad+\left(\int_{0}^{1}|m(t)|\left[\left|f^{\prime \prime}(a)\right|^{1-t}\left|f^{\prime \prime}(b)\right|^{t}\right]^{q} d t\right)^{\frac{1}{q}}\right\} \\
& =\frac{(b-a)^{2}}{4(12)^{\frac{1}{p}}}\left\{\left(\int_{0}^{1}|m(t)| \cdot\left(\frac{\left|f^{\prime \prime}(b)\right|}{\left|f^{\prime \prime}(a)\right|}\right)^{q t} d t\right)^{\frac{1}{q}}\right. \\
& \left.\quad+\left(\int_{0}^{1}|m(t)| \cdot\left(\frac{f^{\prime \prime}(a) \mid}{\left|f^{\prime \prime}(b)\right|}\right)^{q t} d t\right)^{\frac{1}{q}}\right\}
\end{aligned}
$$

where we have used the fact that

$$
\int_{0}^{1}|m(t)| d t=\int_{0}^{\frac{1}{2}} t^{2} d t+\int_{\frac{1}{2}}^{1}(1-t)^{2} d t=\frac{1}{12}
$$

which completes the proof.

Corollary 3 In Theorem 5 , since $\left(\frac{1}{12}\right)^{\frac{1}{p}}<1$, for $p>1$, we have the following inequality:

$$
\left|\frac{1}{b-a} \int_{a}^{b} f(x) d x-f\left(\frac{a+b}{2}\right)\right| \leq \frac{(b-a)^{2}}{4}\left\{\left|f^{\prime \prime}(a)\right| \cdot A(t, q)+\left|f^{\prime \prime}(b)\right| \cdot B(t, q)\right\} .
$$

\section{Results for $(\alpha, m)$-geometrically convex functions}

Theorem 6 Let $f:[0, b] \rightarrow \mathbb{R}_{+}$be a twice differentiable function, $a \in[0, b]$ and $f^{\prime \prime} \in$ $L_{1}[a, b]$. If $\left|f^{\prime \prime}\right|^{q}$ is $(\alpha, m)$-geometrically convex and monotonically decreasing on $[a, b]$ for $b \geq 1, p>1,(\alpha, m) \in(0,1]^{2}$ and $t \in(0,1]$, we have

$$
\left|\frac{f(a)+f(b)}{2}-\frac{1}{b-a} \int_{a}^{b} f(x) d x\right| \leq \frac{(b-a)^{2}}{2}\left(\frac{2^{-1-2 p} \sqrt{\pi} \Gamma(1+p)}{\Gamma\left(\frac{3}{2}+p\right)}\right)^{\frac{1}{p}}\left|f^{\prime \prime}(b)\right|^{m} M(t, q, \alpha),
$$

where $\frac{1}{p}+\frac{1}{q}=1$ and

$$
M(t, q, \alpha)=\left(\int_{0}^{1}\left[\frac{\left|f^{\prime \prime}(a)\right|}{\left|f^{\prime \prime}(b)\right|^{m}}\right]^{q t^{\alpha}} d t\right)^{\frac{1}{q}} .
$$


Proof From Lemma 2 with the properties of modulus and using the Hölder inequality, we have

$$
\begin{aligned}
& \left|\frac{f(a)+f(b)}{2}-\frac{1}{b-a} \int_{a}^{b} f(x) d x\right| \\
& \quad \leq \frac{(b-a)^{2}}{2} \int_{0}^{1} t(1-t)\left|f^{\prime \prime}(t a+(1-t) b)\right| d t \\
& \quad \leq \frac{(b-a)^{2}}{2}\left(\int_{0}^{1}\left(t-t^{2}\right)^{p} d t\right)^{\frac{1}{p}}\left(\int_{0}^{1}\left|f^{\prime \prime}(t a+(1-t) b)\right|^{q} d t\right)^{\frac{1}{q}} \\
& \quad=\frac{(b-a)^{2}}{2}\left(\frac{2^{-1-2 p} \sqrt{\pi} \Gamma(1+p)}{\Gamma\left(\frac{3}{2}+p\right)}\right)^{\frac{1}{p}}\left(\int_{0}^{1}\left|f^{\prime \prime}(t a+(1-t) b)\right|^{q} d t\right)^{\frac{1}{q}} .
\end{aligned}
$$

Since $\left|f^{\prime \prime}\right|^{q}$ is $(\alpha, m)$-geometrically convex and monotonically decreasing on $[a, b]$, by using (1.2) we obtain

$$
\begin{aligned}
I & =\int_{0}^{1}\left|f^{\prime \prime}(t a+(1-t) b)\right|^{q} d t \\
& \leq \int_{0}^{1}\left|f^{\prime \prime}\left(a^{t} b^{m(1-t)}\right)\right|^{q} d t \\
& \leq \int_{0}^{1}\left[\left|f^{\prime \prime}(a)\right|^{t^{\alpha}}\left|f^{\prime \prime}(b)\right|^{m\left(1-t^{\alpha}\right)}\right]^{q} d t \\
& =\left|f^{\prime \prime}(b)\right|^{m q} \int_{0}^{1}\left(\frac{\left|f^{\prime \prime}(a)\right|}{\left|f^{\prime \prime}(b)\right|^{m}}\right)^{q t^{\alpha}} d t .
\end{aligned}
$$

So, we have

$$
\begin{aligned}
& \left|\frac{f(a)+f(b)}{2}-\frac{1}{b-a} \int_{a}^{b} f(x) d x\right| \\
& \quad \leq \frac{(b-a)^{2}}{2}\left(\frac{2^{-1-2 p} \sqrt{\pi} \Gamma(1+p)}{\Gamma\left(\frac{3}{2}+p\right)}\right)^{\frac{1}{p}}\left(\left|f^{\prime \prime}(b)\right|^{m q} \int_{0}^{1}\left(\frac{\left|f^{\prime \prime}(a)\right|}{\left|f^{\prime \prime}(b)\right|^{m}}\right)^{q t^{\alpha}} d t\right)^{\frac{1}{q}} \\
& \quad=\frac{(b-a)^{2}\left|f^{\prime \prime}(b)\right|^{m}}{2}\left(\frac{2^{-1-2 p} \sqrt{\pi} \Gamma(1+p)}{\Gamma\left(\frac{3}{2}+p\right)}\right)^{\frac{1}{p}}\left(\int_{0}^{1}\left(\frac{\left|f^{\prime \prime}(a)\right|}{\left|f^{\prime \prime}(b)\right|^{m}}\right)^{q t^{\alpha}} d t\right)^{\frac{1}{q}}
\end{aligned}
$$

which completes the proof.

\section{Corollary 4 In Theorem 6 ,}

(i) If $\left|f^{\prime \prime}(a)\right|<\left|f^{\prime \prime}(b)\right|^{m}$, then we get

$$
\begin{aligned}
& \left|\frac{f(a)+f(b)}{2}-\frac{1}{b-a} \int_{a}^{b} f(x) d x\right| \\
& \quad \leq \frac{(b-a)^{2}\left|f^{\prime \prime}(b)\right|^{m}}{2}\left(\frac{2^{-1-2 p} \sqrt{\pi} \Gamma(1+p)}{\Gamma\left(\frac{3}{2}+p\right)}\right)^{\frac{1}{p}}\left(\int_{0}^{1}\left(\frac{\left|f^{\prime \prime}(a)\right|}{\left|f^{\prime \prime}(b)\right|^{m}}\right)^{q t^{\alpha}} d t\right)^{\frac{1}{q}} \\
& \quad \leq \frac{(b-a)^{2}\left|f^{\prime \prime}(b)\right|^{m}}{2}\left(\frac{2^{-1-2 p} \sqrt{\pi} \Gamma(1+p)}{\Gamma\left(\frac{3}{2}+p\right)}\right)^{\frac{1}{p}}\left(\int_{0}^{1}\left(\frac{\left|f^{\prime \prime}(a)\right|}{\left|f^{\prime \prime}(b)\right|^{m}}\right)^{q \alpha t} d t\right)^{\frac{1}{q}}
\end{aligned}
$$




$$
\begin{aligned}
= & \frac{(b-a)^{2}\left|f^{\prime \prime}(b)\right|^{m}}{2}\left(\frac{2^{-1-2 p} \sqrt{\pi} \Gamma(1+p)}{\Gamma\left(\frac{3}{2}+p\right)}\right)^{\frac{1}{p}} \\
& \times\left(\frac{\left|f^{\prime \prime}(a)\right|^{q \alpha}-\left|f^{\prime \prime}(b)\right|^{m q \alpha}}{\left|f^{\prime \prime}(b)\right|^{m q \alpha}\left[\ln \left|f^{\prime \prime}(a)\right|^{q \alpha}-\ln \left|f^{\prime \prime}(b)\right|^{m q \alpha}\right]}\right)^{\frac{1}{q}} \\
= & \frac{(b-a)^{2}\left|f^{\prime \prime}(b)\right|^{m(1-\alpha)}}{2}\left(\frac{2^{-1-2 p} \sqrt{\pi} \Gamma(1+p)}{\Gamma\left(\frac{3}{2}+p\right)}\right)^{\frac{1}{p}}\left(L\left[\left|f^{\prime \prime}(a)\right|^{q \alpha},\left|f^{\prime \prime}(b)\right|^{m q \alpha}\right]\right)^{\frac{1}{q}},
\end{aligned}
$$

where we used the fact that if $0<\mu \leq 1,0<t$ and $\alpha \leq 1$, we have

$$
\mu^{t^{\alpha}} \leq \mu^{\alpha t}
$$

(ii) If $\left|f^{\prime \prime}(a)\right|=\left|f^{\prime \prime}(b)\right|^{m}$, then we get

$$
\begin{aligned}
& \left|\frac{f(a)+f(b)}{2}-\frac{1}{b-a} \int_{a}^{b} f(x) d x\right| \\
& \quad \leq \frac{(b-a)^{2}\left|f^{\prime \prime}(b)\right|^{m}}{2}\left(\frac{2^{-1-2 p} \sqrt{\pi} \Gamma(1+p)}{\Gamma\left(\frac{3}{2}+p\right)}\right)^{\frac{1}{p}} .
\end{aligned}
$$

Theorem 7 Let $f:[0, b] \rightarrow \mathbb{R}_{+}$be a twice differentiable function, $a \in[0, b]$ and $f^{\prime \prime} \in$ $L_{1}[a, b]$. If $\left|f^{\prime \prime}\right|$ and $\left|f^{\prime \prime}\right|^{q}$ are $(\alpha, m)$-geometrically convex and monotonically decreasing on $[a, b]$ for $a, b \geq 1, p>1,(\alpha, m) \in(0,1]^{2}$ and $t \in[0,1]$, we have

$$
\begin{aligned}
\left|\frac{1}{b-a} \int_{a}^{b} f(x) d x-f\left(\frac{a+b}{2}\right)\right| & \\
\leq & \frac{(b-a)^{2}}{16}\left\{\frac{1}{3}\left[\left|f^{\prime \prime}(b)\right|^{m} \int_{0}^{\frac{1}{2}} K d t+\left|f^{\prime \prime}(a)\right|^{m} \int_{0}^{\frac{1}{2}} G d t\right]\right. \\
& \left.+\frac{1}{(4 p+2)^{\frac{1}{p}}}\left[\left|f^{\prime \prime}(b)\right|^{m}\left(\int_{\frac{1}{2}}^{1} K^{q} d t\right)^{\frac{1}{q}}+\left|f^{\prime \prime}(a)\right|^{m}\left(\int_{\frac{1}{2}}^{1} G^{q} d t\right)^{\frac{1}{q}}\right]\right\},
\end{aligned}
$$

where $K=\left(\frac{\left|f^{\prime \prime}(a)\right|}{\left|f^{\prime \prime}(b)\right|^{m}}\right)^{t^{\alpha}}, G=\left(\frac{\left|f^{\prime \prime}(b)\right|}{\left|f^{\prime \prime}(a)\right|^{m}}\right)^{t^{\alpha}}$ and $\frac{1}{p}+\frac{1}{q}=1$.

Proof By using Lemma 3 and the properties of absolute value, we have

$$
\begin{aligned}
& \left|\frac{1}{b-a} \int_{a}^{b} f(x) d x-f\left(\frac{a+b}{2}\right)\right| \\
& \quad \leq \frac{(b-a)^{2}}{4}\left\{\int_{0}^{\frac{1}{2}} t^{2}\left|f^{\prime \prime}(t a+(1-t) b)\right| d t+\int_{0}^{\frac{1}{2}} t^{2}\left|f^{\prime \prime}((1-t) a+t b)\right| d t\right. \\
& \left.\quad+\int_{\frac{1}{2}}^{1}(1-t)^{2}\left|f^{\prime \prime}(t a+(1-t) b)\right| d t+\int_{\frac{1}{2}}^{1}(1-t)^{2}\left|f^{\prime \prime}((1-t) a+t b)\right| d t\right\} \\
& \quad=\frac{(b-a)^{2}}{4}\left\{I_{1}+I_{2}+I_{3}+I_{4}\right\} .
\end{aligned}
$$


Since $t^{2}$ is increasing on $\left[0, \frac{1}{2}\right]$ and $\left|f^{\prime \prime}\right|$ is decreasing, by applying the Chebyshev inequality to $I_{1}$ and $I_{2}$ and by applying the Hölder inequality to $I_{3}$ and $I_{4}$, we get

$$
\begin{aligned}
\left|\frac{1}{b-a} \int_{a}^{b} f(x) d x-f\left(\frac{a+b}{2}\right)\right| & \\
\leq & \frac{(b-a)^{2}}{4}\left\{2 \int_{0}^{\frac{1}{2}} t^{2} d t \int_{0}^{\frac{1}{2}}\left|f^{\prime \prime}(t a+(1-t) b)\right| d t\right. \\
& +2 \int_{0}^{\frac{1}{2}} t^{2} d t \int_{0}^{\frac{1}{2}}\left|f^{\prime \prime}((1-t) a+t b)\right| d t \\
& +\left(\int_{\frac{1}{2}}^{1}(1-t)^{2 p} d t\right)^{\frac{1}{p}}\left(\int_{\frac{1}{2}}^{1}\left|f^{\prime \prime}(t a+(1-t) b)\right|^{q} d t\right)^{\frac{1}{q}} \\
& \left.+\left(\int_{\frac{1}{2}}^{1}(1-t)^{2 p} d t\right)^{\frac{1}{p}}\left(\int_{\frac{1}{2}}^{1}\left|f^{\prime \prime}((1-t) a+t b)\right|^{q} d t\right)^{\frac{1}{q}}\right\} \\
= & \frac{(b-a)^{2}}{4}\left\{\frac{1}{12}\left(\int_{0}^{\frac{1}{2}}\left|f^{\prime \prime}(t a+(1-t) b)\right| d t+\int_{0}^{\frac{1}{2}}\left|f^{\prime \prime}((1-t) a+t b)\right| d t\right)\right. \\
& +\frac{1}{(2 p+1)^{\frac{1}{p}} 2^{\frac{2 p+1}{p}}}\left(\left(\int_{\frac{1}{2}}^{1}\left|f^{\prime \prime}(t a+(1-t) b)\right|^{q} d t\right)^{\frac{1}{q}}\right. \\
& \left.\left.+\left(\int_{\frac{1}{2}}^{1}\left|f^{\prime \prime}((1-t) a+t b)\right|^{q} d t\right)^{\frac{1}{q}}\right)\right\} .
\end{aligned}
$$

As $\left|f^{\prime \prime}\right|$ is decreasing, $\left|f^{\prime \prime}\right|$ and $\left|f^{\prime \prime}\right|^{q}$ are $(\alpha, m)$-geometrically convex, from (1.2) we have

$$
\begin{aligned}
& \left|\frac{1}{b-a} \int_{a}^{b} f(x) d x-f\left(\frac{a+b}{2}\right)\right| \\
& \leq \frac{(b-a)^{2}}{4}\left\{\frac{1}{12}\left(\int_{0}^{\frac{1}{2}}\left|f^{\prime \prime}\left(a^{t} b^{m(1-t)}\right)\right| d t+\int_{0}^{\frac{1}{2}}\left|f^{\prime \prime}\left(a^{m(1-t)} b^{t}\right)\right| d t\right)\right. \\
& +\frac{1}{(2 p+1)^{\frac{1}{p}} 2^{\frac{2 p+1}{p}}}\left(\left(\int_{\frac{1}{2}}^{1}\left|f^{\prime \prime}\left(a^{t} b^{m(1-t)}\right)\right|^{q} d t\right)^{\frac{1}{q}}\right. \\
& \left.\left.+\left(\int_{\frac{1}{2}}^{1}\left|f^{\prime \prime}\left(a^{m(1-t)} b^{t}\right)\right|^{q} d t\right)^{\frac{1}{q}}\right)\right\} \\
& \leq \frac{(b-a)^{2}}{4}\left\{\frac{1}{12}\left(\int_{0}^{\frac{1}{2}}\left|f^{\prime \prime}(a)\right|^{t^{\alpha}}\left|f^{\prime \prime}(b)\right|^{m\left(1-t^{\alpha}\right)} d t+\int_{0}^{\frac{1}{2}}\left|f^{\prime \prime}(a)\right|^{m\left(1-t^{\alpha}\right)}\left|f^{\prime \prime}(b)\right|^{t^{\alpha}} d t\right)\right. \\
& +\frac{1}{(2 p+1)^{\frac{1}{p}} 2^{\frac{2 p+1}{p}}}\left[\left(\int_{\frac{1}{2}}^{1}\left[\left|f^{\prime \prime}(a)\right|^{t^{\alpha}}\left|f^{\prime \prime}(b)\right|^{m\left(1-t^{\alpha}\right)}\right]^{q} d t\right)^{\frac{1}{q}}\right. \\
& \left.\left.+\left(\int_{\frac{1}{2}}^{1}\left[\left|f^{\prime \prime}(a)\right|^{m\left(1-t^{\alpha}\right)}\left|f^{\prime \prime}(b)\right|^{t^{\alpha}}\right]^{q} d t\right)^{\frac{1}{q}}\right]\right\}
\end{aligned}
$$

which is the desired result. 
Özdemir et al. Journal of Inequalities and Applications 2014, 2014:180

Page 11 of 12

http://www.journalofinequalitiesandapplications.com/content/2014/1/180

Corollary 5 Under the conditions of Theorem 7, if we choose $f^{\prime \prime}$ is symmetric about $\frac{a+b}{2}$, then we have

$$
\begin{aligned}
& \left|\frac{1}{b-a} \int_{a}^{b} f(x) d x-f\left(\frac{a+b}{2}\right)\right| \\
& \quad \leq \frac{(b-a)^{2}\left|f^{\prime \prime}(b)\right|^{m}}{8}\left\{\frac{1}{3} \int_{0}^{\frac{1}{2}} K d t+\frac{1}{(4 p+2)^{\frac{1}{p}}}\left(\int_{\frac{1}{2}}^{1} K^{q} d t\right)^{\frac{1}{q}}\right\} .
\end{aligned}
$$

Proof From (3.1) and by using the symmetric property of $f^{\prime \prime}$, we get

$$
\begin{aligned}
& \left|\frac{1}{b-a} \int_{a}^{b} f(x) d x-f\left(\frac{a+b}{2}\right)\right| \\
& \leq \frac{(b-a)^{2}}{4}\left\{\frac{1}{12}\left(\int_{0}^{\frac{1}{2}}\left|f^{\prime \prime}(t a+(1-t) b)\right| d t+\int_{0}^{\frac{1}{2}}\left|f^{\prime \prime}((1-t) a+t b)\right| d t\right)\right. \\
& +\frac{1}{(2 p+1)^{\frac{1}{p}} 2^{\frac{2 p+1}{p}}}\left(\left(\int_{\frac{1}{2}}^{1}\left|f^{\prime \prime}(t a+(1-t) b)\right|^{q} d t\right)^{\frac{1}{q}}\right. \\
& \left.\left.+\left(\int_{\frac{1}{2}}^{1}\left|f^{\prime \prime}((1-t) a+t b)\right|^{q} d t\right)^{\frac{1}{q}}\right)\right\} \\
& =\frac{(b-a)^{2}}{4}\left\{\frac{1}{6} \int_{0}^{\frac{1}{2}}\left|f^{\prime \prime}(t a+(1-t) b)\right| d t\right. \\
& \left.+\frac{2}{(2 p+1)^{\frac{1}{p}} 2^{\frac{2 p+1}{p}}}\left(\int_{\frac{1}{2}}^{1}\left|f^{\prime \prime}(t a+(1-t) b)\right|^{q} d t\right)^{\frac{1}{q}}\right\} \\
& =\frac{(b-a)^{2}}{8}\left\{\frac{1}{3} \int_{0}^{\frac{1}{2}}\left|f^{\prime \prime}(t a+(1-t) b)\right| d t\right. \\
& \left.+\frac{1}{(2 p+1)^{\frac{1}{p}} 2^{\frac{1}{p}}}\left(\int_{\frac{1}{2}}^{1}\left|f^{\prime \prime}(t a+(1-t) b)\right|^{q} d t\right)^{\frac{1}{q}}\right\} .
\end{aligned}
$$

By using $(\alpha, m)$-geometric convexity of $\left|f^{\prime \prime}\right|$ and $\left|f^{\prime \prime}\right|^{q}$ and similar calculations, we get

$$
\begin{aligned}
\mid & \frac{1}{b-a} \int_{a}^{b} f(x) d x-f\left(\frac{a+b}{2}\right) \mid \\
\leq & \frac{(b-a)^{2}}{8}\left\{\frac{1}{3} \int_{0}^{\frac{1}{2}}\left|f^{\prime \prime}\left(a^{t} b^{m(1-t)}\right)\right| d t+\frac{1}{(2 p+1)^{\frac{1}{p}} 2^{\frac{1}{p}}}\left(\int_{\frac{1}{2}}^{1}\left|f^{\prime \prime}\left(a^{t} b^{m(1-t)}\right)\right|^{q} d t\right)^{\frac{1}{q}}\right\} \\
\leq & \frac{(b-a)^{2}}{8}\left\{\frac{1}{3} \int_{0}^{\frac{1}{2}}\left[\left|f^{\prime \prime}(a)\right|^{t^{\alpha}}\left|f^{\prime \prime}(b)\right|^{m\left(1-t^{\alpha}\right)}\right] d t\right. \\
& \left.+\frac{1}{(4 p+2)^{\frac{1}{p}}}\left(\int_{\frac{1}{2}}^{1}\left[\left|f^{\prime \prime}(a)\right|^{t^{\alpha}}\left|f^{\prime \prime}(b)\right|^{m\left(1-t^{\alpha}\right)}\right]^{q} d t\right)^{\frac{1}{q}}\right\} \\
= & \frac{(b-a)^{2}\left|f^{\prime \prime}(b)\right|^{m}}{8}\left\{\frac{1}{3} \int_{0}^{\frac{1}{2}}\left(\frac{\left|f^{\prime \prime}(a)\right|}{\left|f^{\prime \prime}(b)\right|^{m}}\right)^{t^{\alpha}} d t+\frac{1}{(4 p+2)^{\frac{1}{p}}}\left(\int_{\frac{1}{2}}^{1}\left(\frac{\left|f^{\prime \prime}(a)\right|}{\left|f^{\prime \prime}(b)\right|^{m}}\right)^{q t^{\alpha}} d t\right)^{\frac{1}{q}}\right\} .
\end{aligned}
$$

So, the proof is completed. 
Competing interests

The authors declare that they have no competing interests.

\section{Authors' contributions}

ÇY and MG carried out the design of the study and performed the analysis. MEÖ participated in its design and coordination. All authors read and approved the final manuscript.

\section{Author details}

${ }^{1}$ Department of Mathematics, K. K. Education Faculty, Atatürk University, Campus, Erzurum, 25240, Turkey. ${ }^{2}$ Graduate School of Natural and Applied Sciences, Ağri Ibrahim Çeçen University, Ağri, Turkey.

Received: 20 September 2013 Accepted: 11 April 2014 Published: 12 May 2014

\section{References}

1. Mitrinovič, DS, Lackovič, IB: Hermite and convexity. Aequ. Math. 28, 229-232 (1985)

2. Beckenbach, EF: Convex functions. Bull. Am. Math. Soc. 54, 439-460 (1948)

3. Jensen, JLWV: On konvexe funktioner og uligheder mellem middlvaerdier. Nyt Tidsskr. Math. 16, 49-69 (1905)

4. Miheşan, VG: A generalization of convexity. In: Proceedings of the Seminar on Functional Equations, Approximation and Convexity. Cluj-Napoca, Romania (1993)

5. Zhang, T-Y, Ji, A-P, Qi, F: On integral inequalities of Hermite-Hadamard type for s-geometrically convex functions. Abstr. Appl. Anal. (2012). doi:10.1155/2012/560586

6. Xi, B-Y, Bai, R-F, Qi, F: Hermite-Hadamard type inequalities for the $m$ - and $(\alpha, m)$-geometrically convex functions. Aequ. Math. (2012). doi:10.1007/s00010-011-0114-x

7. Zhang, X-M: Geometrically Convex Functions, p. $65,78,89,97,108$. An'hui University Press, Hefei (2004) (in Chinese)

8. Özdemir, ME: Inequalities on Geometrically Convex Functions. arXiv:1312.7725v1

9. Özdemir, ME, Yıldı, Ç: New Ostrowski type inequalities for geometrically convex functions. Int. J. Mod. Math. Sci. 8(1), 27-35 (2013)

10. Mitrinović, DS, Pečarić, JE, Fink, AM: Classical and New Inequalities in Analysis. xviii+740 pp. Kluwer Academic, Dordrecht (1993)

11. Alomari, M, Darus, M, Dragomir, SS: New inequalities of Hermite-Hadamard type for functions whose second derivatives absolute values are quasi-convex. Tamkang J. Math. 41(4), 353-359 (2010)

12. Sarıkaya, MZ, Sağlam, A, Yıldıım, H: New inequalities of Hermite-Hadamard type for functions whose second derivatives absolute values are convex and quasi-convex. Int. J. Open Probl. Comput. Sci. Math. 5(3), 1-14 (2012)

10.1186/1029-242X-2014-180

Cite this article as: Özdemir et al.: A note on geometrically convex functions. Journal of Inequalities and Applications 2014, 2014:180

\section{Submit your manuscript to a SpringerOpen ${ }^{\ominus}$ journal and benefit from:}

- Convenient online submission

Rigorous peer review

- Immediate publication on acceptance

Open access: articles freely available online

- High visibility within the field

- Retaining the copyright to your article 